\title{
A new operating mode in experiments searching for free neutron-antineutron oscillations based on coherent neu- tron and antineutron mirror reflections
}

\author{
V.V. Nesvizhevsky ${ }^{1, *}$, V. Gudkov ${ }^{2}$, K.V. Protasov $^{3}$, W.M. Snow ${ }^{4}$, and A.Yu. Voronin ${ }^{5}$ \\ ${ }^{1}$ Institut Max von Laue - Paul Langevin, 71 avenue des Martyrs, Grenoble, France-38000 \\ ${ }^{2}$ Department of Physics and Astronomy, University of South California, South California, USA-29208 \\ ${ }^{3}$ Laboratoire de Phisique Subatomique et de Cosmologie, UGA-CNRS/IN2P3, Grenoble, France-38026 \\ ${ }^{4}$ Department of Physics, Indiana University, 727 E. Third St., Bloomingston, Indiana, USA-47405 \\ ${ }^{5}$ Lebedev Physical Institute, 53 Leninsky prospect, Moscow, Russia-119991
}

\begin{abstract}
An observation of neutron-antineutron oscillations $(n-\bar{n})$, which violate both $B$ and $B-L$ by 2 units, would constitute a fundamental discovery and contribute to our understanding of the baryon asymmetry of the universe. A sufficiently stringent upper constraint on this process would also make a major contribution by ruling out the possibility of post-sphaleron baryogenesis (PSB) involving first-generation quarks, which would mean that sphaleron transitions at the electroweak scale are essential for baryogenesis within the Sakharov paradigm. We show that one can design an experiment with free $n$ using existing or projected neutron sources that can reach the sensitivity needed to rule out PSB if one allows the $n$ and $\bar{n}$, with sufficiently small tangential velocity, to coherently reflect from $n / \bar{n}$ mirrors composed of certain nuclei. We show that the sensitivity of a future experiment can be greatly improved, and a more compact and less expensive apparatus can be realized. A sensitivity gain of $\sim 10^{4}$ in the oscillation probability relative to the existing free- $n$ limit can be reached if one is willing to adopt a long flight path with a horizontal guide viewing a cold neutron source, or a significantly shorter flight path with a vertical guide viewing a very cold neutron source.
\end{abstract}

\section{Introduction}

The possible existence of neutron-antineutron oscillations $(n-\bar{n})$ is of fundamental interest for particle physics and cosmology. $n-\bar{n}$ would violate baryon number by 2 units $(\Delta B=2)$ and an observation possesses many implications for new physics [1-38].

The possibility to conduct a much more sensitive search for $\Delta B=2$ violating processes, especially with $\Delta(B-L)=2$, such as $n-\bar{n}$ oscillations, has started to attract more attention both from theory and experiment. The latter processes are important for cosmological arguments, which use the well-known Sakharov criteria [2] to generate the baryon asymmetry of the universe starting from a $B=0$ initial condition. A baryon asymmetry generated in the early universe from $B$-violating processes, which come from new physics above the electroweak phase transition and conserves $B-L$, such as the simplest $\Delta B=1$ and $\Delta L=1$

*e-mail: nesvizhevsky@ill.eu 
proton decay $p \rightarrow \pi_{0} e^{+}$, could be erased at the electroweak phase transition by sphalerons. These thermally-activated transitions of nonperturbative electroweak gauge fields with nontrivial winding number violate both $B$ and $L$ separately but conserve $B-L$ and can therefore erase a preexisting $B$ asymmetry by converting it into a $L$ asymmetry, which is much harder to confront with astrophysical observations given our ignorance of the contribution to the net $L$ of the universe from the very large number of neutrinos. Over the last few years, more theoretical models have been proposed which posses $\Delta B=2$ processes which can lead to $n-\bar{n}$ oscillations without giving $p$ decay [23-26, 29, 30, 34, 35, 38]. Thus, an interesting class of models can give the baryon asymmetry in a process called post-sphaleron baryogenesis (PSB). The PSB model space is constrained both by the present limits on $n-\bar{n}$ oscillations combined with the lack of observations of its characteristic decay channels so far at the LHC [35]. The type of experiment proposed in this work has the capacity to rule out this and related PSB models operating in the light quark sector in combination with additional constraints on other consequences of the model from the LHC. Any set of experiments which are able to rule out PSB would be of fundamental importance for cosmology as in this case one would be able to conclude that an understanding of the electroweak sphaleron transitions in the early universe is necessity for understanding the baryon asymmetry within the Sakharov paradigm. New analysis of existing data to constrain $\Delta B=2$ processes have appeared from SuperK [39] and SNO [40]. The possibility of more sensitive searches for $\Delta B=2$ processes in future large underground detectors such as HyperK [41] and Dune [42] is leading to new theoretical work on $\bar{n} A$ dynamics [43]. The connection between $\Delta B=2$ processes and CPT/Lotentz violation in the well known Standard Model Extension [44] has been analysed [45]. An observation of $n-\bar{n}$ oscillations would put stringent limits on CPT violation in the nucleon sector also on long-range gauge fields coupled to $B-L$, where it would improve on present constraints from tests of the equivalence principle over a broad range of couplings and ranges [46, 47]. Several theoretical studies [48-52] have clarified the subtleties involved in properly understanding the discrete symmetry transformations of a composite strongly interacting bound system like the $n$. Related studies make it clear that the observation of $n-\bar{n}$ oscillations would indicate that $n$ is in fact a Majorana particle [53]. A new $\Delta B=2$ physical process, $n-\bar{n}$ conversion, has been identified and described theoretically [54], and the possibility of neutron - mirror neutron oscillations $[55,56]$, indicated as a possibility in UCN experiments [57-60] is the subject of active investigation [61]. Finally recent studies have investigated in greater depth the limits of the so-called quasifree condition for the evolution of the $n / \bar{n}$ amplitudes described below [62]. These developments in theory and experiment show that the subject of $\Delta B=2$ processes has become more interesting and that therefore the experimental approach described in this paper is of general interest to the physics community.

Experimental searches for $\Delta B=2$ processes involving $n$ have so far been conducted in two ways. One channel searches for $n-\bar{n}$ oscillations of free $n$ in a setup where one tries to avoid all possible Standard Model interactions of the $n$ and the $\bar{n}$ they can oscillate into. One arranges the $n$ to prevent touching matter and to shield external magnetic fields, as both effects will shift the relative energy between $n$ and $\bar{n}$ states by an amount $\Delta E$. Despite the fact that this energy shift is much larger than the off-diagonal mixing term $\varepsilon$ in the effective Hamiltonian for the $n / \bar{n}$ two state system, it does not greatly suppress the oscillation rate if the observation time of the system is short compared to $\Delta E / \hbar$ (with $\hbar$ the reduced Planck constant). In this so-called quasifree regime, the relative phase shift between the $n$ and $\bar{n}$ states from the time development, $e^{-\Delta E t / \hbar}$, is small enough that the oscillation probability still growth quadratically with the observation time. Since a search for the appearance of an $\bar{n}$ in a free $n$ beam can be conducted in very low background conditions, the interpretation of an upper bound of this process is clearly defined and does not require any further modeling of the system. The other channel searches for $n-\bar{n}$ oscillations of the $n$ bound in nuclei. Here one 
needs a model to calculate the degree of suppression of the rate in the presence of a very large $\Delta E[9,63-68]$. Although this suppression in the dense nuclear environment is very large, the very large number of $n$, which can be observed in low-background underground detectors of large volume, makes this a sensitive search mode. Now, underground experiments give slightly better limits compared to free $n$ searches when analyzed using the existing models of $n-\bar{n}$ oscillations in nuclei and of the distribution of the reaction products of $\bar{n} A$ annihilation. However, there are at least two issues, which are important to understand about the limits from these searches. One is that the interpretation of the results is dependent on models of the $\bar{n}$ annihilation in heavy nuclei. These are optical models, which are constrained by several types of measurements, including $\bar{p}$ and $\bar{n}$ interactions in nuclei. The data analysis needed to extract bounds from these experiments also depends on the details of an event generator, which can accurately describe the many different reaction products from the $\bar{n}$ annihilation in nuclei. The simplifications of the physics in this event generator introduce additional uncertainties in the interpretation of the inferred limit through its influence on the detailed experimental signature in the detector as these detectors all have significant backgrounds in the annihilation reaction channels. In addition, the bounds from these experiments are not physically equivalent to those from free $n$ since there are additional $\Delta B=2$ processes which can happen inside a nucleus which cannot happen for free $n$. Therefore, strictly speaking, the bounds from these two $\Delta B=2$ processes should be treated as complementary.

We analyse a new version of $n-\bar{n}$ experiment using slow $n$ : an (almost) free $n$ oscillation search in which we allow the $n / \bar{n}$ to bounce of $n / \bar{n}$ optical mirrors. We show that, over a broad fraction of phase space acceptance of a $n / \bar{n}$ guide and for certain choices for the nuclei composing the guide material, the probability of coherent reflection of $n / \bar{n}$ from the guide walls is quite high, the relative phase shift between $n$ and $\bar{n}$ is quite low, and the precision of theoretical estimations of these parameters is sufficiently high to provide relatively small uncertainties in the calculation of the experimental sensitivity. Furthermore, recent advances in the effective field theory (EFT) treatment of $\bar{n} A$ interactions make it possible to improve the precision and to quantify the residual errors of such an analysis. Below, we show that an experiment conducted under these conditions relaxes some of the important constraints on the free $n$ oscillation searches and in principle allows us to achieve a much higher sensitivity. This approach preserves the very low background conditions that have already been achieved in free $n$ oscillation searches and does not require the same level of detail in the model of the annihilation products needed to interpret the underground detector annihilation experiments.

An expression for the probability of $n-\bar{n}$ oscillations, which takes into account $n \beta$-decay and $\bar{n}$ annihilation in medium, is found for instance in ref. [69]. If one starts with a pure $|n\rangle$ state at $t=0$, then there is a finite probability for it to evolve to an $|\bar{n}\rangle$ at $t \neq 0$ given by

$$
P_{n \rightarrow \bar{n}}(t) \approx \frac{\varepsilon^{2}}{\omega^{2}+\left(\frac{\Gamma_{\alpha}}{2}\right)^{2}} e^{-\Gamma_{\beta} t}\left(1+e^{-\Gamma_{\alpha} t}-2 e^{\frac{-\Gamma_{\alpha} t}{2}} \cos (\omega t)\right),
$$

where $\Gamma_{\beta}$ and $\Gamma_{\alpha}$ are the $n \beta$-decay and $\bar{n}$ annihilation widths, $E_{n}$ and $E_{\bar{n}}$ are the energy of $n$ and $\bar{n}$, and $\varepsilon=E_{n}-E_{\bar{n}}$ the mixing parameter. This equation resembles the well known equation for the probability of neutral kaon oscillations. For any practical observation time, $e^{-\Gamma_{\beta} t} \approx 1$ and $\omega t \ll 1$, and the expression for the probability of oscillations is reduced to

$$
P_{n \rightarrow \bar{n}}(t) \approx \varepsilon^{2} e^{-\frac{\Gamma \alpha t}{2}} t^{2}
$$

After differentiating eq. (2), we get the optimum observation time

$$
t_{0}=\frac{4}{\Gamma_{\alpha}}
$$


Provided condition (3), the $n-\bar{n}$ oscillation probability is

$$
P_{n \rightarrow \bar{n}} \approx 2.1\left(\frac{\varepsilon}{\Gamma_{\alpha}}\right)^{2}
$$

Neglecting the widths, these equations reduce to the known quasifree limit $P_{n \rightarrow \bar{n}} \approx\left(t / \tau_{n \rightarrow \bar{n}}\right)^{2}$, where $\tau_{n \rightarrow \bar{n}}=1 /|\varepsilon|$ (for natural units $\hbar=c=1$ ) is the characteristic time of oscillations. As a few annihilation events suffice for a positive signal (provided backgrounds are low), the figure of merit in such an experiment with the total number $N$ of observed $n$ is $F \approx N t^{2}$.

The best constrain for $\tau_{n \rightarrow \bar{n}}$ using free $n$ comes from the state-of-the-art experiment [70] with cold $n$ passing through a long vacuum tube shielded against external magnetic fields; earlier experiments are described in [71, 72]. An ambitious project at a projected fundamental physics beamline at ESS [73] proposes an analogous scheme with advanced parameters to increase the sensitivity by a factor of $G \approx 10^{2}-10^{3}$. In particular, it would profit from a large solid angle of extraction of $n$ from the ESS source, thus form a large total $n$ flux. These $n$ have to be focused to an annihilation detector to increase the statistical sensitivity. We argue that the sensitivity can be increased further if one allows the reflection of $n / \bar{n}$ from $n / \bar{n}$ guide walls, thus increasing significantly the attainable length of the experiment, the observation time $t$, and the statistical accuracy. Some literature on the subject gives the incorrect impression that the coherence of the mixed $n / \bar{n}$ amplitude is always destroyed upon contact with matter. This is not true for a coherent $n$ reflection from a surface. As long as the $n$ is not "observed" (annihilated) and the phase difference between $n$ and $\bar{n}$ components of the amplitude upon reflection is small enough, the sensitivity for the $\bar{n}$ component continues to grow with time just as it does in the quasifree limit. We give a general formalism and provide estimations for an experiment at the PF1B beam at the Institute Laue-Langevin [74] as an example. Greater sensitivity could be achieved at other neutron sources/guides. Extraction of $n$ directly from a $n$ source through a large solid angle, as projected in [73], might bring a large improvement. A sensitivity can be precisely calculated for any particular configuration and $n$ source using the formalism developed in this article and standard $n$ optical calculations. The primary $n$ source brightness is one key parameter, which defines the experimental sensitivity, but not the only one. The observation time and, therefore, the experiment length is another key parameter. Space constraints (in particular, the maximum $n-\bar{n}$ guide length achievable) are quite important factors. The total $n$ flux is important; a large source with medium brightness could be more suitable than a small-size $n$ source with record brightness. The mean velocity of $n$ is another key parameter; a softer $n$ spectrum with the same flux can constrain $\tau_{n-\bar{n}}$ as well as an experiment with faster $n$ and a proportionally larger length.

We describe the interaction of $n$ and $\bar{n}$ with walls in Section 2, taking into account the dominant phenomena: $\bar{n}$ annihilation and phase shift between $n$ and $\bar{n}$ upon wall reflection. We consider a specific experimental scheme in Section 3. In Section 4, we estimate the sensitivity of such an experiment and mention alternative geometries and $n$ spectra, which might also be employed using these ideas. The new developments in our understanding of low-energy $\bar{n}$ interactions in nuclei are summarized in Section 6.

\section{Reflection of neutrons and antineutrons from walls}

Already 4 years after the discovery of neutrons by James Chadwick [75], Enrico Fermi understood that coherent scattering of $n$ with wavelengths large compared with the nuclear size can be described in terms of an effective neutron-matter optical potential [76]. The scattering amplitude can be formally obtained using perturbation theory with a point-like pseudo-potential (to note his priority, this potential is often called Fermi potential): $U(\vec{r})=\left(\left(2 \pi \hbar^{2}\right) / m\right) b \delta \vec{r} r$, 
with $m$ the reduced $n$ mass and $b$ the complex neutron-nucleus $(n A)$ scattering length. Then, coherent interaction of $n$ with matter is described using a complex uniform optical potential $U(r)=\left(\left(2 \pi \hbar^{2}\right) / m\right)(\rho / \mu) b$, with $\rho$ the mass density of material, and $\mu$ the atomic mass. If a material consists of different nuclei, the value of its optical potential is the sum of all partial contributions. The values of $n$ optical potential for many nuclei have been measured with $1 \%$ accuracy or better. If a transversal energy of $n$ is smaller than the real part of optical potential of the surface material, such $n$ is reflected. This property is an important tool in neutron research, which allows the construction of neutron guides [77]. If the total $n$ energy is smaller than the real part of optical potential, the $n$ is reflected at any incidence angle. Fedor Shapiro's group in Dubna [78] and Albert Steyerl in Munich [79] discovered such neutrons (Ultra Cold Neutrons, or UCNs). Since then numerous experiments use their main property: (nearly) total elastic reflection from walls. Below we neglect the small $n$ loss probability of typically $10^{-4}-10^{-5}$, caused by their nuclear absorption and inelastic scattering. As noted as early as in 1980, one can also use UCNs for constraining $\tau_{n-\bar{n}}[8,12,80,81]$.

Nobody has measured the reflection of $\bar{n}$ from matter because of the absence of sufficient slow $\bar{n}$ fluxes. Nevertheless, the same approach can be applied to $n$ and $\bar{n}$ keeping in mind that imaginary parts of the optical potential for $\bar{n}$ are always large due to $\bar{n}$ annihilation. Crucial parameters for the analysis of this problem are the probability of $n$ and $\bar{n}$ reflection per wall collision ( $\rho_{n}$ and $\rho_{\bar{n}}$, respectively) and the difference of phase shifts of the $n$ and $\bar{n}$ amplitude components per wall collision $\Delta \varphi_{n \bar{n}}=\varphi_{n}-\varphi_{\bar{n}}$, where $\varphi_{n}$ and $\varphi_{\bar{n}}$ are phase shifts for $n$ and $\bar{n}$, respectively. $\rho_{n}, \rho_{\bar{n}}, \varphi_{n}, \varphi_{\bar{n}}$ depend on the energy of $n / \bar{n}$ transversal motion $\left(e_{n}\right.$ and $e_{\bar{n}}$, respectively) and on the optical potentials of the wall material $U_{n}=V_{n}+i W_{n}$ and $U_{\bar{n}}=V_{\bar{n}}+i W_{\bar{n}}$ for the $n$ and $\bar{n}$, respectively, where $V_{n}$ and $V_{\bar{n}}$ are real parts of the optical potential, and $W_{n}$ and $W_{\bar{n}}$ are the corresponding imaginary parts. We denote $e_{h o r}$ and $e_{v e r}$ as the energies corresponding to horizontal and vertical components of motion (equal for $n$ and $\bar{n}$ in vacuum). We also consider only specular reflection of $n / \bar{n}$ from walls since the probability of non-specular reflection from a well polished surface of a $n$ guide is typically as low as $\sim 10^{-3}$ even for UCNs [82, 83]. Let's consider $n / \bar{n}$ with energy $e=\left(k^{2}\right) /(2 m)$ of transverse motion to be small compared to the real parts of the optical potentials,

$$
e \ll V_{n}, e \ll V_{\bar{n}}
$$

but comparable to the value of imaginary part for $\bar{n}$,

$$
e \sim W_{\bar{n}}
$$

and we assume (consistent with the theoretical analysis of the $\bar{n} A$ data for various nuclei) optical potentials with imaginary parts much smaller than the real parts,

$$
W_{n} \ll V_{n}, W_{\bar{n}} \ll V_{\bar{n}},
$$

and imaginary parts for $n$ significantly lower than imaginary parts for $\bar{n}$,

$$
W_{n} \ll W_{\bar{n}} .
$$

These conditions $(5,6,7,8)$ do not affect the generality of our estimations and conclusions. In fact, conditions $(5,6)$ correspond to long lifetimes of $\bar{n}$ in a $n / \bar{n}$ guide and thus will be the goal of our approach. The long lifetimes correspond to small angular divergencies in the $n / \bar{n}$ beam achieved by proper shaping of $n / \bar{n}$ angular distributions in the first part of the $n / \bar{n}$ guide. The condition (7) assumes an evident requirement of large real parts of optical potentials and small imaginary parts. It is met with a good accuracy for all cases of practical interest. Although we aim at both imaginary parts (for $n$ and $\bar{n}$ ) to be small, evidently this 
goal can be easier achieved for $n$, thus we assume the condition (8). By condition (8), the probability of $n$ reflection is close to unity: $\rho_{n}=1$, and the probability of $\bar{n}$ reflection is:

$$
\begin{array}{r}
\rho_{\bar{n}}=1-\frac{4 k k_{\bar{n}}^{\prime \prime}}{\left(k+k_{\bar{n}}^{\prime \prime}\right)^{2}+\left(k_{\bar{n}}^{\prime}\right)^{2}}, k_{\bar{n}}^{\prime}-i k_{\bar{n}}^{\prime \prime}=\sqrt{2 m\left(V_{\bar{n}}-i W_{\bar{n}}-e\right)}, \\
k_{\bar{n}}^{\prime}=\sqrt{m\left(\sqrt{\left(V_{\bar{n}}-e\right)^{2}+\left(W_{\bar{n}}\right)^{2}}+\left(V_{\bar{n}}-e\right)\right)}, k_{\bar{n}}^{\prime \prime}=\sqrt{m\left(\sqrt{\left(V_{\bar{n}}-e\right)^{2}+\left(W_{\bar{n}}\right)^{2}}-\left(V_{\bar{n}}-e\right)\right)},
\end{array}
$$

with $k_{\bar{n}}$ the complex momentum of $\bar{n}$ inside the wall. It is instructive to estimate the value of the reflection coefficient (9) for 3 cases: $\left|V_{\bar{n}}\right| \gg\left|W_{\bar{n}}\right|$ (weak absorption), $\left|V_{\bar{n}}\right| \sim\left|W_{\bar{n}}\right|$ (comparable real and imaginary parts of the optical potential), and $\left|V_{\bar{n}}\right| \ll\left|W_{\bar{n}}\right|$ (strong absorption). In ALL cases, the reflection probability of $\bar{n}$ is close to unity and quite insensitive to the variation in the magnitude of the $\bar{n}$ optical potential coming from theoretical analysis of $\bar{n}$ data. It is also consistent with known facts for analogous physical systems where the presence of a large imaginary part of the optical potential does not destroy quantum coherence. A simple example is the reflection of polarized light from a metal mirror. Using conditions $(5,6,7,8)$, we simplify eq. (9) as follows: $k_{\bar{n}}^{\prime} \approx \sqrt{2 m V_{\bar{n}}}, k_{\bar{n}}^{\prime \prime} \approx \sqrt{m\left(W_{\bar{n}}^{2} /\left(2 V_{\bar{n}}\right)\right)}$, and thus a first order approximation for the probability of $\bar{n}$ annihilation, providing conditions $(5,6,7,8)$ are met, is

$$
1-\rho_{\bar{n}} \approx \frac{4 k k_{\bar{n}}^{\prime \prime}}{\left(k_{\bar{n}}^{\prime}\right)^{2}}
$$

The phase shifts (providing conditions $(5,6,7,8)$ are met) from one reflection are respectively

$$
\Delta \varphi_{n \bar{n}} \approx \frac{2 k}{k_{n} k_{\bar{n}}^{\prime}}\left(k_{n}-k_{\bar{n}}^{\prime}\right), \Delta \varphi_{n}=\arctan \left(-\frac{2 k k_{n}}{k^{2}-k_{n}^{2}}\right), \Delta \varphi_{\bar{n}}=\arctan \left(-\frac{2 k k_{\bar{n}}^{\prime}}{k^{2}-\left(k_{\bar{n}}^{\prime}\right)^{2}-\left(k_{\bar{n}}^{\prime \prime}\right)^{2}}\right) .
$$

Considering low-energy $\bar{n} A$ interaction when $\bar{n}$ de Broglie wavelength is larger than an interatomic distance, the $\bar{n} A$ scattering can be described by a complex length $b_{\bar{n} A}$. The similarity between $\bar{p}$ and $\bar{n}$ low-energy scattering on nuclei allowed the authors of [84] to suggest the simple fitting formula for the scattering length based on the analysis of $\bar{p} A$ data:

$$
b_{\bar{p}(\bar{n}) A}=\left(1.54 A^{1 / 3}-i 1.0\right) f m,
$$

with $A$ the atomic number. The imaginary part of $b_{\bar{p}(\bar{n}) A}(\sim 1 \mathrm{fm})$ is determined by the diffusive tail of an effective $\bar{n} A$ potential that is approximately the same for all nuclei. The real part is proportional to the nuclei linear size. $\bar{n} A$ low-energy scattering is insensitive to details of nuclei structure due to intense annihilation of $\bar{n}[84,85]$. The knowledge of scattering lengths $b_{\bar{n}} A$ allows calculating optical potentials of $\bar{n} A$ interaction and lifetimes of $\bar{n}$ on a horizontal surface in the gravitational field, as described above. Table 1 presents these values for some materials; using eq. (13), the reader can easily perform calculations for other elements/isotopes/materials ${ }^{1}$. The smooth dependence of $b_{\bar{n} A}$ values on atomic number $A$ indicates a similarity between low-energy $\bar{n}$ and $n$ scattering, where potential scattering plays a dominant role for most nuclei. Thus formula (13) can be considered as a good approximation for the calculations of average low-energy $\bar{n}$ scattering lengths. This phenomenon was noticed in work [87], and was used in [88] for constraining fundamental short-range forces. The weak dependence of $b_{\bar{n} A}$ on nuclei simplifies the choice of certain isotope/element composition of a material, which would give equal real parts of $U_{n}$ and $U_{\bar{n}}$. As only one of numerous

\footnotetext{
${ }^{1}$ Note that the value of $\bar{n} C u$ optical potential published in $[69,86]$ is inconsistent with the corresponding scattering length, published in the same paper, and is probably wrong
} 


\begin{tabular}{|c|l|l|l|}
\hline Element & $b_{\bar{n} A}[\mathrm{fm}]$ & $U_{\bar{n}}[\mathrm{neV}]$ & $\tau_{\bar{n}}[\mathrm{~s}]$ \\
\hline $\mathrm{C}$ & $3.5-i$ & $103-i 29$ & 1.7 \\
\hline $\mathrm{Mg}$ & $3.5-i$ & $39-i 11$ & 1.0 \\
\hline $\mathrm{Si}$ & $3.7-i$ & $48-i 13$ & 1.2 \\
\hline $\mathrm{Ni}$ & $4.7-i$ & $111-i 24$ & 2.3 \\
\hline $\mathrm{Cu}$ & $4.7-i$ & $104-i 22$ & 2.2 \\
\hline $\mathrm{Zr}$ & $5.3-i$ & $59-i 11$ & 1.8 \\
\hline $\mathrm{Mo}$ & $5.3-i$ & $89-i 16$ & 2.3 \\
\hline $\mathrm{W}$ & $6.5-i$ & $106-i 16$ & 3.0 \\
\hline $\mathrm{Pb}$ & $6.7-i$ & $57-i 8.6$ & 2.3 \\
\hline $\mathrm{Bi}$ & $6.7-i$ & $49-i 7$ & 2.1 \\
\hline
\end{tabular}

Table 1. Parameters, which characterize the interaction of $\bar{n}$ with different materials: $b_{\bar{n} A}(\bar{n} A$ the scattering length), $U_{\bar{n}}$ (the complex optical potential for this material), $\tau_{\bar{n}}$ (the time of storage of $\bar{n}$ with close-to-zero vertical energy on a horizontal surface in the Earth's gravitational field). Calculations for all elements are averaged over isotope compositions.

examples, the isotopic composition ${ }^{184} W(87.7 \%)+{ }^{186} W(12.3 \%)$ provides real parts of the optical potential for $n$ and $\bar{n}$ both equal 106neV.

In Section 4, we will show how such a choice of materials enhances the sensitivity of $n-\bar{n}$ experiments. In Section 3, we will introduce an example experimental geometry, calculate the frequency of wall collisions, lifetimes, and sensitivity to $n-\bar{n}$ oscillations.

\section{A scheme for the experiment using a slow neutron guide}

Consider a ballistic neutron guide [74] consisting of two parts. Its cross sectional area at the upstream section, which the $n$ from the source first encounter, increases gradually along its length. The $h$ be its height and $d$ its width at the entrance, $H$ its height and $D$ its width at the exit, and $l$ the length of the first part. In the second part of the ballistic guide, the cross sectional area is constant over its length with dimensions $H$ and $D$ and the length $L$. Since the $n$, which strikes the wall in the extending part of the guide, see the wall recede in their frame, these collisions lower transverse components of the $n$ velocity as the beam moves through the guide. The diverging guide section is designed so that vertical and horizontal velocity components are mixed. Collisions with the walls in the second part of the guide with constant cross sectional area preserve the transverse velocity components. We denote $s=h d$ the cross sectional area of the first section at its entrance (typically, $\sim 10^{2} \mathrm{~cm}^{2}$ ), and $S=H D$ its cross sectional area at the exit (typically, $\sim 10^{4} \mathrm{~cm}^{2}$ ). We assume that the perpendicular velocity components of the $n$ entering the ballistic guide are $<2 v_{c r i t}^{N i}$, where $v_{c r i t}^{N i} \approx 7 \mathrm{~m} / \mathrm{s}$ is the critical velocity of $\mathrm{Ni}$. In accordance with Liouville's theorem, the transverse velocities at the exit of a properly designed ballistic guide, in which the diverging part of the $n$ guide adiabatically narrows the transverse beam phase space, will be restricted by $\left|v_{\text {hor }}\right| \cdot\left|v_{\text {vert }}\right|<\left(2 v_{\text {crit }}\right)^{2} \frac{d h}{D H}$. A typical high value of $U_{\bar{n}}$ in Table (1) is $>100 \mathrm{neV}$ that corresponds to critical velocities of $>4 \mathrm{~m} / \mathrm{s}$. In order to analyse the problem in the low-energy limit of transverse velocities, we assume that the mean transversal velocity is at least 4 times lower than that, or $\overline{\left|v_{\text {hor }}\right|} \sim \overline{\left|v_{\text {vert }}\right|} \sim$ $1 \mathrm{~m} / \mathrm{s}$. These 2 conditions are met, if the guide cross section is expanded by at least the factor of $\frac{D H}{d h} \sim 49$. Due to the gravity, $n / \bar{n}$ with too low vertical energy would not reach the upper wall of the guide. Thus, its vertical expansion is constrained. As the Liouville condition involves the factor $(D H) /(d h)$, not $D$ and $H$ separately, an elliptical divergent section would 
allow decreasing $H$ and increasing $D$. To avoid unpractical guide cross sections with $D \gg H$, one might use a few superimposed flat guides. To take into account this option, we will use a relatively small value of $D=1 \mathrm{~m}$ and show that the interaction of $\bar{n}$ with horizontal walls would not limit the sensitivity of $n-\bar{n}$ experiments even then. Thus, a $n / \bar{n}$ beam can be shaped so that perpendicular velocities are small and the loss in statistics is also small.

A ballistic $n / \bar{n}$ guide of any shape can be simulated for any $n$ distribution at the entrance. Note that the diverging guide section also contributes to the $n-\bar{n}$ sensitivity as the $n / \bar{n}$ incidence angles are relatively small. We selected copper as a material for this analysis because the real part of its optical potential for $\bar{n}$ is large, the imaginary part is relatively small, and $C u$ has been used in the past for $n$ mirrors with success. Another example is tungsten with a specially selected isotopic composition mentioned above as it provides larger storage time of $\bar{n}$ on the surface. The search for a better material is an important task.

Due to the effect of gravity, we will consider the interaction of $n / \bar{n}$ with horizontal and vertical guide walls separately. The frequencies of $n / \bar{n}$ collisions with horizontal walls and bottom (provided $n / \bar{n}$ never touch the top wall), $f_{\text {hor }}$ and $f_{\text {vert }}$ respectively, are

$$
f_{\text {hor }}=\frac{\left|v_{\text {hor }}\right|}{D}, f_{\text {vert }}=\frac{g}{2\left|v_{\text {vert }}\right|} \text {. }
$$

The $\bar{n}$ lifetimes associated with side-walls and bottom, $\tau_{h o r}^{\rho, \bar{n}}$ and $\tau_{\text {vert }}^{\rho, \bar{n}}$ respectively, are:

$$
\tau_{h o r}^{\rho, \bar{n}}=\frac{1}{f_{h o r}\left(1-\rho_{\bar{n}}\right)}, \tau_{\text {vert }}^{\rho, \bar{n}}=\frac{1}{f_{\text {vert }}\left(1-\rho_{\bar{n}}\right)},
$$

and the annihilation probability is given by eq. (9). A characteristic lifetime for the accumulation of a phase shift equal 1 radian between $n$ and $\bar{n}$ amplitudes associated with side-wall and bottom collisions, $\tau_{\text {hor }}^{\Delta \varphi, \bar{n}}$ and $\tau_{\text {vert }}^{\Delta \varphi, \bar{n}}$ respectively, are

$$
\tau_{h o r}^{\Delta \varphi, \bar{n}}=\frac{1}{f_{h o r} \Delta \varphi_{n \bar{n}}}, \tau_{v e r t}^{\Delta \varphi, \bar{n}}=\frac{1}{f_{\text {vert }} \Delta \varphi_{n \bar{n}}},
$$

and the phase shift is given by eq. (12).

In Section 4, we will discuss the choice of experimental parameters and estimate the $n-\bar{n}$ experiment sensitivity. As usual for free $n$ experiments, we assume that an $\bar{n}$ annihilation foil is located downstream. An annihilation detector around the foil measures the $\bar{n}$ component of the amplitude arriving at the foil. $\bar{n}$, which annihilate upstream in the $n / \bar{n}$ guide, would not be detected unless very close to the foil, and we neglect their contribution to the signal.

\section{Estimation of sensitivity of $n-\bar{n}$ oscillation experiment}

The characteristic accumulation time for a phase difference $\Delta \varphi_{n \bar{n}}$ of $1 \mathrm{rad}$ between $n$ and $\bar{n}$ amplitude due to the side-walls collisions (eqs. $(12,14,16)$ ),

$$
\tau_{\text {hor }}^{\Delta \varphi, \bar{n}}=\frac{D}{\left|v_{\text {hor }}\right|} \frac{\sqrt{V_{n} V_{\bar{n}}}}{2 \sqrt{\overline{\bar{e}_{\text {hor }}}} \cdot\left|\sqrt{V_{n}}-\sqrt{V_{\bar{n}}}\right|},
$$

is $32 s$ for $C u$ and very long for isotopically adjusted ${ }^{184+186} W$. In both cases, it is longer than the practical observation time in the apparatus for cold $n / \bar{n}$ beam experiment. We therefore neglect this effect. The characteristic accumulation time for a phase difference $\Delta \varphi_{n \bar{n}}$ of $1 \mathrm{rad}$ between $n$ and $\bar{n}$ amplitudes due to bottom collisions,

$$
\tau_{\text {vert }}^{\Delta \varphi, \bar{n}}=\frac{\overline{\left|v_{\text {vert }}\right|}}{g} \frac{\sqrt{V_{n} V_{\bar{n}}}}{\sqrt{\overline{e_{\text {vert }}}} \cdot\left|\sqrt{V_{n}}-\sqrt{V_{\bar{n}}}\right|},
$$


is $2.2 s$ for $C u$ and long for ${ }^{184+186} W . \tau_{v e r t}^{\Delta \varphi, \bar{n}}$ for $C u$ is an order of magnitude smaller than $\tau_{h o r}^{\Delta \varphi, \bar{n}}$ because of the effect of gravity on trajectories. Nevertheless, a proper mixture of materials/isotopes for the $n / \bar{n}$ guide walls (as done for ${ }^{184+186} W$ ) would increase $\tau_{\text {vert }}^{\Delta \varphi, \bar{n}}$ considerably due to the small term $\left(\left(\sqrt{V_{n}}-\sqrt{V_{\bar{n}}}\right) \rightarrow 0\right)$ in the denominator of eqs. $(17,18)$. We therefore believe this effect will not limit the sensitivity of experiments on $n-\bar{n}$ oscillations as well.

The annihilation time due to the side-wall collisions (eqs. $(11,14,15))$,

$$
\tau_{h o r}^{\rho, \bar{n}}=\frac{D}{2 \mid \overline{v_{h o r} \mid}} \frac{\left(V_{\bar{n}}\right)^{3 / 2}}{W_{\bar{n}} \sqrt{\overline{e_{h o r}}}},
$$

is $11 s$ for $C u$ and $15 s$ for ${ }^{184+186} W$. These values are also large enough to be neglected for now. Finally, the annihilation time due the bottom collisions,

$$
\tau_{\text {vert }}^{\rho, \bar{n}}=\frac{\overline{\left|v_{\text {vert }}\right|}}{g} \frac{\left(V_{\bar{n}}\right)^{3 / 2}}{W_{\bar{n}} \sqrt{\overline{e_{\text {vert }}}}},
$$

is $2.2 s$ for $C u$ and $3.1 s$ for ${ }^{184+186} W$. Not surprisingly, annihilation of $\bar{n}$ in the accumulated reflections from the bottom of the guide is the main limiting factor. Due to the gravity, such collisions cannot be avoided in a horizontal geometry. $\tau_{v e r t}^{\rho, \bar{n}}$ does not depend on the energy of vertical motion in the low-energy limit (note the term $\overline{\left|v_{\text {vert }}\right|} / \sqrt{\overline{e_{v e r t}}}$ and the fact that we separate two dimensionless terms in eq. (20) to help the reader to perform numerical estimations). This weak energy dependence justifies our approach of estimating all values in the low-energy limit neglecting energy corrections. This is due to the compensation of two effects: 1 . the larger frequency of bounces of $n / \bar{n}$ for lower energy of vertical motion, 2 . the smaller probability of $\bar{n}$ annihilation per bounce for lower energy of vertical motion. This observation simplifies considerably an analysis of any experimental configuration: we can deal with a single number (eq. (20)), which defines the overall lifetime; other parameters are less relevant for a properly designed experiment in the horizontal geometry. In addition, this observation allows us to make a simple estimate of the impact of uncertainties in the calculation of the $\bar{n}$ optical potential of the mirror material on the experimental results. As the annihilation time $\tau_{\text {vert }}^{\rho, \bar{n}}$ is proportional to the inverse imaginary part of optical potential for $\bar{n}$ (eq. (20)), a typical $10-20 \%$ error in the estimation of $W_{\bar{n}}$ gives only a $10-20 \%$ error in the lifetime estimation. If the observation time is shorter than the optimum value (eq. (3)), then the impact would be accordingly even smaller. The impact of uncertainty in the real part of the optical potential is negligible by comparison.

The above arguments treat $n / \bar{n}$ motion semiclassically. However, the results coincide with quantum expressions $[89,90]$ in the low-energy limit. The scattering amplitude of a cold $n / \bar{n}$ on a nucleus, in terms of the scattering length, is $a=1 / k_{n, \bar{n}}=1 / \sqrt{2 m\left(V_{n, \bar{n}}+W_{n, \bar{n}}\right)}$ [90]. The effective horizontal momentum of a $n / \bar{n}$ in a box with a size $D$ is $k \approx \pi j /\left(D-2 / k_{n, \bar{n}}\right)$, where $j$ is quantum number of the box-like state. The corresponding horizonal energy levels shift due to the interaction with wall is $\Delta E_{h o r} \approx 4 \varepsilon_{h o r} a / D=4 \varepsilon_{h o r} /\left(D k_{n, \bar{n}}\right)$. For vertical motion, the energy levels shift due to the interaction with bottom surface is $\Delta E_{v e r}=m g a=m g / k_{n, \bar{n}}$ [90]. This expression is energy-independent and is consistent with the arguments given above based on semiclassical $n / \bar{n}$ motion. It is easy to check that the fully quantum expressions for the characteristic timescales $\tau_{\text {hor }}^{\Delta \varphi, \bar{n}}=1 / \omega_{\text {hor }}, \tau_{\text {hor }}^{\rho, \bar{n}}=1 / \Gamma_{a, h o r}, \tau_{\text {ver }}^{\Delta \varphi, \bar{n}}=1 / \omega_{\text {ver }}, \tau_{\text {ver }}^{\rho, \bar{n}}=1 / \Gamma_{a, v e r}$ also coincide with the semiclassical expressions obtained above.

The gravitational constraints on $n-\bar{n}$ oscillation experiment from wall reflections are obviously no longer relevant for a vertical $n / \bar{n}$ guide. While gravity affects only slightly cold neutrons (CNs) in a vertical $n / \bar{n}$ guide, it dramatically modifies the motion of very cold neutrons (VCNs), which are defined by energies in the range of microelectronVolts. Consider 
an upwards-directed fountain of VCNs. Due to gravity, the observation time is long and can include both the rise and the fall. Annihilation and phase shifts in the guide walls are low and can be neglected. To compensate lower VCN than CNs fluxes available, one could design a large-surface VCN source based on fluorinated nanodiamond reflectors [91, 92]. For a characteristic VCN velocity of $\sim 50 \mathrm{~m} / \mathrm{s}$, the fountain height is $\sim 125 \mathrm{~m}$ and the observation time is $\sim 10 \mathrm{~s}$. For a characteristic flux density of $\sim 10^{7}-10^{8} \mathrm{VCN} / \mathrm{cm}^{2} / \mathrm{s}$, we approach the sensitivity achievable with horizontally extracted CNs.

The effect of gravity on $n-\bar{n}$ oscillation experiments can also in principle be partly suppressed by replacing a horizontal straight guide considered above by a parabolic $n / \bar{n}$ guide. It could be designed to follow a trajectory of a $n / \bar{n}$ with some mean velocity in the distribution from the cold $n$ source. Then we can replace $g$ in eq. (20) by an effective smaller $g_{\text {eff }}$ value, and $\tau_{v e r}^{\rho, \bar{n}}$ will increase reversely. A conservative estimation, for the $n$ flux characteristics as at $\mathrm{PF} 1 \mathrm{~B}$, is the following. Compare the factors of merit or the experiment considered above and the best existing result [70]. The total number of $n$ at PF1 was $3 \cdot 10^{18}$. The total number of $n$ at PF1B per year would be a factor of 4-5 larger; it will increase by a few times if the experiment runs longer. A gain factor due to the increased observation time, would be $\sim 10^{2}$ for $t \sim 1 \mathrm{~s}$, and $\sim 10^{4}$ for $t \sim 10 \mathrm{~s}$. As the main gain is associated with a huge increase in the experiment length, any project has to optimize the sensitivity relative to the $n / \bar{n}$ beam geometry, $n$ spectrum, the budget and spatial constrains; such considerations are beyond the scope of this paper. Probably a wise approach would be to start developing the experiment from a short version, to demonstrate its performance, and then increase the experiment length.

The reduction of mean $n$ velocity is of major importance for this experiment. This argument strongly motivates the development of better VCN sources. Measurements with UCNs of existing densities are not yet competitive, however compact, cheap and might be a first step towards more sensitive experiments. The whole analysis in this paper was initiated by the observation that while the annihilation time is short for $n / \bar{n}$ transverse energies close to the real part of the optical potential [86], it is longer for smaller energies. However, even the limit of gravitational quantum states of neutrons [93] does not bring an additional improvement in the observation time as the annihilation time saturates at the lowest energies.

Neglecting factors of order of unity and assuming that detection of a couple of annihilation events in a background-free experiment means the observation of $n / \bar{n}$ oscillations, one can estimate the overall accuracy to be equal

$$
\tau_{n-\bar{n}} \sim \frac{\sqrt{F T}}{\Gamma_{a}},
$$

where $\tau_{n-\bar{n}}$ is the time of $n-\bar{n}$ oscillations, $F$ the total $n$ flux, and $T$ the duration of experiment. While a maximum $F$ is provided by $\mathrm{CNs}$, an optimum $\mathrm{CN}$ guide is long. One might prefer a softer $n$ spectrum to take full advantage of this operation mode. Any particular experiment configuration can be easily simulated using formalism given above, without reducing it to the low-energy limit.

\section{Recent theoretical developments in low-energy antineutron-nucleus interactions}

Recent progress in our understanding of the $\bar{n} A$ optical potential based on the analysis of new data on $p \bar{p}$ production from heavy meson decay and $e^{+} e^{-}$annihilation would enable us to bound the size of the relative phase shifts of the $n$ and $\bar{n}$ amplitudes and $\bar{n}$ annihilation rates. We briefly summarize here the new related developments. For more details, see [94]. 
Since the analysis of antinucleon interactions presented in [84], new information on $\bar{p}$ interactions from the threshold behavior of decays of heavy mesons to $p \bar{p}$ and from $e^{+} e^{-}$ annihilation to $p \bar{p}$ now more tightly constrain the $p \bar{p}$ interaction [95-98]. New phenomenological $N N$ potential models have been published based on input from this data $[99,100]$, and an update of the Nijmegen partial wave analysis (PWA) of $p \bar{p}$ scattering data has also been presented [101]. The G-parity symmetry of the low-energy strong interactions, a combination of charge conjugation and a rotation in isospin space, is generally expected in QCD to be obeyed at the $\sim 1 \%$ of accuracy. It directly relates the component of $N \bar{N}$ potential from pion exchange to the $N N$ potential, which is very well determined from decades of data on $N N$ scattering. Thus, the $N \bar{N}$ potential is fixed by the data on the $N N$ interaction combined with the underlying chiral symmetry of pion-nucleon dynamics. With the development of chiral EFT of the $N N$ interaction, which incorporate by construction all of the low-energy symmetries of QCD and allow in principle for the calculation of systematic higher-order corrections to calculations of $N N$ processes once a sufficient number of low-energy constants are determined from experiment, one can now make a more direct connection between measurements of low-energy $N N$ interactions and QCD. Chiral EFTs of the $N$ interaction have been extensively developed, and recently an analysis of the $N N$ interaction to order N3LO has appeared [94], which explicitly treats the annihilation cross section data in such a way that the optical theorem is satisfied. In this work, the EFT parameters are determined from high energy data, and then one can compare them directly with the low-energy data from the hyperfine structure of $p \bar{p}$ and from low-energy $p \bar{p}$ annihilation. These EFT predictions are in good agreement with experiment. In fact, the overall agreement between $N \bar{N}$ data and theory is almost as good as that for $N N$ data. This achievement means that we now have a good understanding of the $\bar{n} A$ interaction.

Recent EFT calculations of $\bar{n} A$ interactions [94] can now describe antinucleon scattering phase shifts with good accuracy. These new results confirm previous theoretical estimates of antinucleon optical potentials. The key quantitative importance of this recent achievement is that such an EFT realizes by construction all of the symmetries of the strong interaction between nucleons and antinucleons and therefore in principle fully embodies the low-energy limit of QCD. It can also be realized as a perturbative extension in the power of $p / \lambda$, where $p$ is a typical momentum between the $n$ and the nucleons, and $\lambda$ is the QCD scale. It therefore in principle possess quantifiable errors. Furthermore, the $n$ and $\bar{n}$ kinetic energies are small compared to $\lambda$ and the interaction is dominated by $s$-wave scattering as determined by the phase shifts. The dependence of the real and imaginary $n$ and $\bar{n}$ optical potentials in matter on the nuclear properties is limited to two key pieces of information: the coherent scattering length (which is the specific linear combination of $n A$ scattering amplitudes which leaves the states of the matter unchanged) and the total $n A$ cross section, which combined with unitarity determines the imaginary part of the $n$ optical potential through the optical theorem of scattering theory. The uncertainties in the $n$ (and more importantly, the $\bar{n}$ ) optical potentials can therefore in principle be quantified in a rigorous, model-independent way, which allows one to determine both the sensitivities of a $n-\bar{n}$ oscillation experiment and the uncertainties of the nuclear physics.

We can also anticipate new experimental data, which will be useful to further constrain our knowledge of $\bar{n} A$ properties relevant for our conclusions. New knowledge of antinucleon interactions will come from the FAIR facility under construction at GSI. Further experimental precision on the properties of the $n$ skin of nuclei, which is helpful to improve our knowledge of the nuclear surface properties, which dominate the $\bar{n} A$ scattering length physics, is anticipated from measurements of the $n$ skin of heavy nuclei using parity violating electron scattering at Jefferson Lab. We therefore anticipate reductions in the present uncertainties of $\bar{n} A$ interactions as a by-product of these other measurements. 


\section{Conclusion}

We propose a new operating mode for advanced searches for $n-\bar{n}$ oscillations using coherent $n$ and $\bar{n}$ mirror reflection. Its advantages can include higher sensitivity, smaller transverse beam sizes, longer observation times, and lower costs. $\bar{n}$ annihilation and different phaseshifts for $n$ and $\bar{n}$ at their reflection from surface limits the sensitivity of such experiments, however we have found practical operating regimes for which both the $\bar{n}$ annihilation probability is relatively small, the coherence between $n$ and $\bar{n}$ amplitudes is preserved for an extended time, and the quantum-mechanical evolution of the $n / \bar{n}$ superposition amplitude can be predicted with a good accuracy and can satisfy the quasifree condition. For the practical examples we discuss above, the $\bar{n}$ component of the amplitude can propagate without annihilating and without large phase shifts relative to the $n$ component for up to $\sim 10 \mathrm{~s}$. The estimated increase in sensitivity of such an experiment over the best current limit can reach 4 orders of magnitude in terms of the oscillation probability. Such a gain corresponds to characteristic oscillation times of $\tau_{n-\bar{n}} \sim 10^{10} \mathrm{~s}$. For horizontal extraction of $n$, the optimum geometry of $n / \bar{n}$ guide is a parabola which follows the trajectory of a $n / \bar{n}$ with an effective mean transverse velocity. For vertical extraction of slower $n$ (a $n$ fountain-style $n-\bar{n}$ experiment), guides could be used to confine the sideways dimensions of the fountain with negligible impact to sensitivity. In both cases (horizontal and vertical), however, the gain in sensitivity is associated with largely increasing the length of setup, thus a reasonable compromise should be searched for.

The authors are grateful to the participants of INT-17-69W Workshop "Neutronantineutron oscillations: appearance, disappearance and baryogenesis" in Seattle, USA, held on 23-27 October 2017, as well as to our colleagues from GRANIT collaboration. V.G. is grateful for support to the U.S. Department of Energy, Office of Science, Office of Nuclear Physics program under Award Number DE-SC0015882. W.M.S. acknowledges support from NSF PHY-1614545 and from the Indiana University Center for Spacetime and Symmetries.

\section{References}

[1] Stueckelberg, E.C.G., Helv. Phys. Acta 11, 312 (1938)

[2] Sakharov, A.D., JETP. Lett. 5, 24 (1967)

[3] Kuzmin, V.A., JETP. Lett. 13, 335 (1970)

[4] Georgi, H., et al., Phys. Rev. Lett. 32, 438 (1974)

[5] Zeldovich, Ya.B., Phys. Lett. A 59, 254 (1976)

[6] Hooft, G.t', Phys. Rev. D 14, 3432 (1978)

[7] Mohapatra, R.N., et al., Phys. Rev. Lett. 44, 1316 (1980)

[8] Kazarnovskii, M.V., et al., JETP Lett. 32, 82 (1980)

[9] Kuo, T.K., et al., Phys. Rev. Lett. 45, 93 (1980)

[10] Chang, L.N., et al., Phys. Lett. B 92, 103 (1980)

[11] Mohapatra, R.N., et al., Phys. Lett. B 94, 183 (1980)

[12] Chetyrkin, K.G., et al., Phys. Lett. B 99, 358 (1981)

[13] Dolgov, A.D., et al., Rev. Mod. Phys. 53, 1 (1981)

[14] Cowsik, R., et al., Phys. Lett. B 101, 237 (1981)

[15] Rao, S., et al., Phys. Lett. B 116, 238 (1982)

[16] Misra, S.P., et al., Phys. Rev. D 28, 249 (1983)

[17] Rao, S., et al., Nucl. Phys. B 232, 143 (1984)

[18] Kuzmin, V.A., et al., Phys. Lett. B 155, 36 (1985) 
[19] Fukugita, M., et al., Phys. Lett. B 174, 45 (1986)

[20] Shaposhnikov, M.E., Nucl. Phys. B 287, 757 (1987)

[21] Dolgov, A.D., Phys. Rep. 222, 309 (1992)

[22] Huber, S.J., et al., Phys. Lett. B 512, 365 (2001)

[23] Babu, K.S., et al., Phys. Lett. B 508, 269 (2001)

[24] Nussinov, S., et al., Phys. Rev. Lett. 88, 171601 (2002)

[25] Babu, K.S., et al., Phys. Lett. B 518, 269 (2006)

[26] Dutta, B., et al., Phys. Rev. Lett. 96, 061801 (2006)

[27] Berezhiani, Z., et al., Phys. Rev. Lett. 96, 081801 (2006)

[28] Bambi, C., et al., Nucl. Phys. B 763, 91 (2007)

[29] Babu, K.S., et al., Phys. Rev. D 79, 015017 (2009)

[30] Mohapatra, R.N., J. Phys. G 36, 104006 (2009)

[31] Dolgov, A.D., Phys. At. Nucl. 73, 558 (2010)

[32] Gu, P.-H., Phys. Lett. B 705, 170 (2011)

[33] Morrissey, D.E., et al., New J. Phys. 14, 125003 (2012)

[34] Arnold, J.M., et al., Phys. Rev. D 87, 075004 (2013)

[35] Babu, K.S., et al., Phys. Rev. D 87, 115019 (2013)

[36] Canetti, L., et al., Phys. Rev. D 87, 093006 (2013)

[37] de Gouvea, A., et al., Phys. Rev. D 90, 016011 (2014)

[38] Berezhiani, Z., Europ. J. Phys. C 76, 705 (2016)

[39] Gustafson, J., et al., Phys. Rev. D 91, 072009 (2016)

[40] Aharmim, B., et al., Phys. Rev. D 96, 092005 (2016)

[41] HyperKamiokande Proto-Collaboration, ArXiv:1805.04163 (2018)

[42] Dune Collaboration, ArXiv:1307.7335 (2014)

[43] Golubeva, E.S., et al., ArXiv:1804.10270 (2018)

[44] Kostelesky, V.A., et al., Rev. Mod. Phys. 83, 11 (2011)

[45] Babu, K.S., et al., Phys. Rev. D 91, 096009 (2015)

[46] Mohapatra, R.N., Phys. Rev. D 94, 054034 (2016)

[47] Kamyshkov, Yu., Europ. J. Phys. C 77, 301 (2017)

[48] Berezhiani, Z., et al., ArXiv:1506.05096 (2015)

[49] Gardner, S., et al., Phys. Rev. D 91, 096010 (2015)

[50] Gardner, S., et al., Phys. Rev. D 93, 096008 (2016)

[51] Fujikawa, K., et al., Phys. Rev. D 94, 115009 (2016)

[52] McKeen, D., et al., Phys. Rev. D 94, 076002 (2016)

[53] Babu, K.S., et al., Phys. Rev. D 91, 013008 (2015)

[54] Gardner, S., et al., Phys. Rev. D 97, 056008 (2018)

[55] Berezhiani, Z., Int. J. Mod. Phys. 19, 3775 (2004)

[56] Berezhiani, Z., et al., Phys. Rev. Lett. 96, 081801 (2006)

[57] Serebrov, A.P., et al., Phys. Lett. B 663, 181 (2008)

[58] Serebrov, A.P., et al., Nucl. Instr. Meth. A 611, 137 (2009)

[59] Altarev, I., et al., Phys. Rev. D 80, 032003 (2009)

[60] Berezhiani, Z., et al., Europ. Phys. J. C 72, 1974 (2012)

[61] Berezhiani, Z., et al., Phys. Rev. D 96, 035039 (2017)

[62] Young, A., Phys. Rev. D 95, 036004 (2017)

[63] Dover, C.C., et al., Phys. Rev. D 27, 1090 (1983)

[64] Dover, C.B., et al., Nucl. Instr. Meth. A 284, 13 (1989) 
[65] Gal, A., Phys. Rev. C 61, 028201 (2000)

[66] Chung, J., et al., Phys. Rev. D 66, 032004 (2002)

[67] Friedman, E., et al., Phys. Rev. D 78, 16002 (2008)

[68] Abe, K., et al., Phys. Rev. D 91, 072006 (2015)

[69] Kerbikov, B.O., Phys. At. Nucl. 66, 2228 (2003)

[70] Baldo-Ceolin, M., et al., Zeit. Phys. C 63, 409 (1994)

[71] Fidecaro, G., et al., Phys. Lett. B 156, 122 (1985)

[72] Bressi, G., et al., Nouvo Cem. 5, 731 (1990)

[73] Phillips, D.G., et al., ArXiv:1410.1100 (2015)

[74] Abele, H., et al., Nucl. Instr. Meth. A 562, 407 (2006)

[75] Chadwick, J., Nature 129, 312 (1932)

[76] Fermi, E., La Ric. Sient. 7, 13 (1936)

[77] Maier-Leibnitz, H., J. Nucl. En. 17, 217 (1963)

[78] Luschikov, V.I., et al, JETP Lett. 9, 23 (1969)

[79] Steyerl, A., Phys. Lett. B 29, 33 (1969)

[80] Yoshiki, H., et al, Nucl. Phys. A 501, 869 (1989)

[81] Yoshiki, H., et al, Nucl. Phys. A 536, 648 (1992)

[82] Nesvizhevsky, V.V., Nucl. Instr. Meth. A 557, 576 (2006)

[83] Nesvizhevsky, V.V., et al., Nucl. Instr. Meth. A 578, 435 (2007)

[84] Batty, B.O., et al., Nucl. Phys. A 689, 721 (2001)

[85] Protasov, K.V., et al., Europ. Phys. J. C 8, 429 (2000)

[86] Kerbikov, B.O., et al., JETP 98, 417 (2004)

[87] Peshkin, M., et al., Am. J. Phys. 39, 324 (1971)

[88] Nesvizhevsky, V.V., et al., Phys. Rev. D 77, 034020 (2008)

[89] Voronin, A.Yu., et al., Phys. Rev. D 73, 044029 (2006)

[90] Voronin, A.Yu., et al., Phys. Rev. A 83, 032903 (2011)

[91] Nesvizhevsky, V.V., et al., Carbon 130, 799 (2018)

[92] Nesvizhevsky, V.V., et al., Phys. Rev. A 97, 023629 (2018)

[93] Nesvizhevsky, V.V., et al., Nature 415, 297 (2002)

[94] Dai, L.-Y., et al., J. High En. Phys. 7, 078 (2017)

[95] Bai, J.Z., et al. (BES Collaboration), Phys. Rev. Lett. 91, 022001 (2003)

[96] Aubert, B., et al. (BaBar Collaboration), Phys. Rev. D 72, 051101 (2005)

[97] Aubert, B., et al. (BaBar Collaboration), Phys. Rev. D 73, 012005 (2006)

[98] Ablikim, M., et al. (BES IIICollaboration), Phys. Rev. Lett. 108, 112003 (2012)

[99] Entem, D.R., et al., Phys. Rev. C 73, 045214 (2006)

[100] El-Bennich, B., et al., Phys. Rev. C 79, 054001 (2009)

[101] Zhou, D., et al., Phys. Rev. C 86, 044003 (2012) 